\title{
Multi-Axis Retarder Arrays by Masked Oblique Deposition
}

\author{
M. D. Arnold, I. J. Hodgkinson, Q. H. Wu \\ MacDiarmid Institute for Advanced Materials and Nanotechnology, Department of Physics, \\ University of Otago, PO Box 56, Dunedin, New Zealand \\ R. J. Blaikie \\ MacDiarmid Institute for Advanced Materials and Nanotechnology, Department of Electrical and \\ Computer Engineering, University of Canterbury, Private Bag 4800, Christchurch, New Zealand
}

\begin{abstract}
We report a technique for single-deposition production of arrays with differently-oriented nano-structured elements, which we optimize for application to form-birefringent retarder arrays. The technique involves masking of oblique physical vapor deposition, manipulating the local availability of vapor angles to create spatially variant properties in the structured coating. We have designed a variety of retarder arrays using symmetry considerations, and constructed a selection based on suitability for spatially-multiplexed complete-Stokes polarimetry. In particular, we emphasize square lattice designs with a two-by-two repeat unit, consisting of three retarders with azimuths at 0,45 and 90 degrees and a zero-retardation element. The silicon retarder arrays were tested using visible-wavelength transmission techniques which confirm the success of the method. Finally, we discuss some considerations for application of the technique to microlithography, and we infer a lateral resolution limit related to the coating thickness.
\end{abstract}




\section{INTRODUCTION}

Obliquely deposited films are porous with oriented internal nano-structure, a consequence of shadowing effects and limited diffusion. Such nano-structured films have mechanical, thermal, electrical, magnetic and optical properties ${ }^{1}$ different to the bulk, resulting in many potential applications ${ }^{2}$ including fluid sensors ${ }^{3}$ and polarization control $^{4}$. A particular property of interest is form birefringence, which is a direct result of the structural anisotropy, and enables production of thin-film polarizing elements, such as retarders ${ }^{1}$, linear ${ }^{5}$ and circular $^{6}$ polarizers.

Recent investigations ${ }^{7-12}$ of obliquely deposited materials have focused on lithographic manipulation, both on micro and on macro scales. A distinction is made relative to the natural structural scale of the coating, and for nanostructured coatings optimized for visible wavelengths the macro-scale can be as small as a few microns. We are particularly interested in the macro-patterning of polarizing elements, with applications including optical lithography, visual displays, and especially imaging polarimetry.

Production of patterned polarizing arrays has previously been achieved, but there are still issues requiring attention. One class of methods involve bulk patterning of innately polarizing materials such as polymers ${ }^{13,14}$, electrochemical porous silicon, and obliquely deposited films ${ }^{8,15}$. A key consideration is that some applications require different film properties in the same device, in the same way that spatially-multiplexed color imaging and display technology requires red, green and blue pixels. In practical terms each desired film property usually requires separate fabrication steps (and possibly additional planarization) which increases the risk of failure. Alternatively, direct writing of subwavelength polarizing structures ${ }^{16,17}$ currently presents additional mass-production challenges, especially for the visible region ${ }^{17,18}$. In this article, we present a general method for patterning obliquely deposited films that avoids some of the aforementioned limitations.

In a deposition process, masks such as the one shown in Figure 1 can be used to affect the orientation of vapor flux available to different areas. At the most basic level, masking is binary amplitude patterning, as utilized in normal liftoff processes. Additionally, masked oblique deposition has been used to control lateral placement of elements ${ }^{19}$ by changing the deposition angle. In the current study, we additionally consider the effect of the strong relationship between vapor angle and film properties. A mask determines the local vapor angles available at different areas of the 
substrate, and hence it is possible to achieve specific spatial distributions of film properties. In the example shown in Figure 1, flux aligned with the direction of a given mask slot passes through and is deposited, whereas flux perpendicular is blocked. The result is that the film structure in a particular area is oriented to the corresponding mask slot, giving the possibility of different film properties in different areas.

\section{DESIGN CONSIDERATIONS}

The process of designing a mask to produce a given set of material properties hinges on two well-studied relationships. One is between the material properties and vapor angles, and the other is between vapor angles and the mask.

The relationship between material properties and vapor angles is extensively described in the literature, but we briefly detail some points relevant to this discussion. The basic layout for oblique vapor deposition is shown in Figure 2. Optimal polarization performance is typically achieved when the elevation angle $\theta$ is approximately $60 \sim 70^{\circ}$, but the various polarizing elements use different regimes of dynamic azimuth $\xi$ to define the normal-incidence optical planes. Retarders can be produced using a single fixed azimuth, but improved performance may be achieved under serial bideposition (SBD), where the azimuth is rapidly stepped in half-turn increments. Linear polarizers can be produced using quarter-wave layers deposited with perpendicular azimuths. Circular polarizers are twisted materials with the vertical periodicity tuned to the desired wavelength, requiring azimuth increments finer than one-sixth-turn. We emphasize that retarders have superior spectral tolerance and the simplest deposition requirements, and hence from this point on we focus on retarder arrays.

Moving now to the effect of the mask, vapor angles are a function of the source (globally) and the mask (locally). Well collimated sources are preferred for this technique, and the effect of the mask is adequately described by geometrical ray-tracing. In terms of achieving a particular pattern given the set of desired angles, one can use reverse ray-tracing to demarcate the possible spatial domain for the mask, but there does not appear to be any direct way to definitively choose which subset the mask should occupy. One could proceed through trial-and-error simulations, but significant simplification is possible by invoking heuristics such as symmetry. 
The target application will likely set the required lattice and shape symmetry, for example typical detector and display arrays have square symmetry. Further, design work is minimized if the azimuthal symmetry of the deposition matches the lattice symmetry. Specifically, matching azimuths to principal lattice vectors allows constant elevation angle $\theta$. Finally, lateral dimensions are related to vertical dimensions by the elevation angle $\theta$. In this paper we emphasize periodic designs, so that the lateral period $p$ is usually exactly related to a vertical period $h$ :

$p=h \tan \theta$

We can apply a similar relationship to the non-periodic example in Figure 1, where $p$ is identified as the upper bound on the width of the mask opening $a$, and $h$ is the mask height.

In addition, the azimuths are likely constrained by the desired film properties. A specific example, applicable to the designs presented here, is imaging polarimetry. We note that it is possible to construct an imaging polarimeter using only variable retarders and any fixed linear polarizer. A general requirement for optimal performance is that the retarder azimuths are different, and the specific detail depends on whether we consider "complete" or "linear-only" polarimeters. Linear-only polarimeters, which require only three unique elements, are conveniently optimized using three-fold symmetry. Complete polarimeters require four filters, but no more than two azimuths may differ by exactly $90^{\circ}$, which precludes the use of pure four-fold symmetry. Three-fold symmetry can be used if one element is left blank, but other solutions give better polarimetry performance. Notably, quasi-four-fold symmetry can be utilized $^{20}$ if we introduce a non-principal lattice azimuth, optimally $45^{\circ}$. Non-principal optical azimuths complicate design because usual practice would dictate a non-principal deposition azimuth, and hence a different elevation angle.

Here we introduce an alternative deposition scheme ${ }^{20}$ for such a non-principal-azimuth (NPA) element. The SBD technique may be adapted by altering the symmetry of the two SBD azimuths, resulting in growth and optical planes bisecting the deposition planes, assuming equal amounts are deposited from each. Specifically, under repeated stepping between two azimuths that are perpendicular, the optical plane lies approximately at $45^{\circ}$. This is distinctly different from the discrete layer case where the retardations would cancel, resulting in no retardation. We find that the effect is optimized nearer glancing elevation, and when $\theta=76^{\circ}$ the retardation of the dual-azimuth NPA element is approximately equal to that of areas receiving only one azimuth (and hence half the amount of material). 
Having described some considerations required for application of the masking technique, we now present some suitable designs, which help to cement the concepts presented so far.

\section{SQUARE MASK DESIGNS}

\section{Contacted Cells}

Looking first at square lattices, a possible type of mask uses thin vertical walls that form square cells, as in Figure 3. A two-by-two periodic arrangement is generated with a pixel pitch is half that of the cell pitch. If discrete depositions are used for each azimuth, regions with two perpendicular overlapping azimuths will have zero net retardation, and an additional $45^{\circ}$ deposition azimuth is required (shown dotted). The $45^{\circ}$ azimuth requires a special elevation angle, $\theta_{45}$, given by projection of the diagonal azimuth onto the square lattice:

$\sqrt{2} p=h \tan \theta_{45}$

Alternatively, SBD of the perpendicular azimuths will generate a $45^{\circ}$ retarder azimuth in the overlap, so no additional azimuths are required in this case and a region is left blank.

\section{Contacted Pillars}

Another type of mask that rests on the substrate consists of square pillars on a square lattice with $50 \%$ duty cycle (see Figure 4). Perpendicular deposition azimuths are applied, and regions of equal and opposite retardation are generated in the gaps directly between pillars. The application of SBD gives $45^{\circ}$ retarder azimuth in the overlap regions diagonally between pillars. Removal of the mask should lift-off any coating in the overlying region, leaving a blank element. Note that that this design has more tolerance than all of the others: specifically the mask height specified by the exact shadowing equation (1) can be exceeded, which will ensure lift-off.

\section{Suspended Sheet}

A mask could be suspended above the substrate, and a two-by-two periodic pattern can be generated using the scheme shown in Figure 5. Note that the deposited elements must lie directly underneath the mask to generate the correct blocking of the perpendicular azimuth. Hence there is no overlap region, and a separate $45^{\circ}$ deposition is required. The advantage of this scheme is that it allows SBD for optimal birefringence, but note that the design continues to work correctly in the absence of SBD. In the design shown, allowance has been made for finite mask thickness as 
required for mechanical stability. Mask thickness is projected to a reduction of the deposited element width $w$ (along the deposition plane), and so the apertures have expanded width $a$ as compensation. Deposition symmetry implies that the mask apertures are symmetrical, which inevitably results in a deviation from the regular element size. The design has been optimized to make the best use of space, so that the elements touch exactly ( $a=2 p-w)$, requiring that:

$d \tan \theta=2(p-w)$

\section{TRIANGULAR MASK DESIGNS}

The basic mask forms have been clearly illustrated by the square designs, but we now turn to alternatives based on three-fold symmetry, to show a broader range of possibilities. These include suspended masks (Figure 6) and contact masks (Figure 7).

\section{Suspended Masks}

The square suspended mask design can be transformed onto a three-fold symmetric lattice by a shearing of the squares to rhombi, as seen in Figure 6a. The advantage of the transformed design (and all other three-fold symmetric designs) is that now all deposition azimuths lie on principal lattice vectors, meaning that elevation angle is constant. In the design shown, we have assumed minimal mask thickness, but appropriate compensation modifications can be applied if thickness is finite (as in Figure 5).

Another three-fold symmetric suspended mask uses a hexagonal lattice. One possible scheme is shown in Figure 6b, where minimal mask thickness is assumed, but naturally distortion of the elements will result when mask thickness is finite.

An alternative suspended hexagonal deposition scheme - Figure 6c - generates only three distinct elements (none blank) under the limit of maximum aperture enlargement of Figure 6b. In this case it is the vertical aspect of the mask that provides the necessary shadowing, requiring that the mask thickness equal the mask-substrate separation. The elements are rhombi which lie under the mask struts, and SBD is allowed but not required.

\section{Contacted Masks}

A contact hexagon mask, as in Figure 7a, can be used to generate rhombi in a similar fashion to the square cell mask. SBD is not used here, as it would result in overlapping regions which appear to be of no advantage. 
A triangular cell mask such as Figure 7b can also be used to generate four unique elements, provided the correct orientation of azimuths is applied. Similarly, SBD is not used, because the odd number of sides would result in a complicated overlapping of the shapes.

We conclude this section by noting that the technique is flexible, and while we have already presented several designs, even more are possible depending on the application. We focus now on proof-of-concept, by experimentally implementing all of our square lattice designs.

\section{EXPERIMENTAL METHODS}

Several square-lattice masks were generated, by one of two methods. The majority of designs were engineered at the millimeter scale into thin aluminum plate, with a printed-circuit-board milling machine. The advantage of this technique was rapid prototyping, convenience of manufacture and analysis, and the ability to re-use masks.

On the other hand, not all designs were appropriate to this method, and target applications require micron scale patterning. The square pillar mask is particularly appropriate to micro-lithographic production, and one such mask was generated using conventional photo-lithography of a positive photoresist (Shipley S1813).

All coatings were deposited in a Leybold-Heraeus 560 system with a stage for inclination and rotation of the substrate, represented diagrammatically in Figure 2. Material was evaporated by e-beam, from a fixed 1cm crucible, and condensed on the substrate at a separation of $25 \mathrm{~cm}$. In this study silicon was evaporated due to superior formbirefringence and process simplicity, but note that the masking technique itself can be applied to any suitable material. Typical operating pressure was approximately $10^{-4}$ mbar. The pressure and geometry used result in a narrow angular distribution. The substrate is generally heated to improve film properties, but care was required when organic material (i.e. photoresist) was involved. Quasi-simultaneous deposition was achieved when required by timemultiplexed (step-wise) substrate rotation. The stepping process is computer controlled, with the transition between the chosen azimuths occurring at small thickness increments signalled by the quartz crystal monitor.

Following film deposition, masks were removed, either by simple physical separation (aluminum masks) or by ultrasonic agitation in acetone (photoresist masks). The removal of the mask is necessary for process completion of some designs and facilitates optical analysis. Polarizing properties of the films were measured, by scanning 
ellipsometry and by polarization imaging. Transmission-mode normal-incidence spatial-scanning ellipsometry was performed at $633 \mathrm{~nm}$ with a rotating-retarder-fixed-polarizer system, giving quantitative retardation information. Visible polarization imaging was performed either using a transmission polarizing microscope, or polarizing filters with a camera. The imaging allowed inspection of pattern integrity and rapid confirmation of polarizing properties. Finally, scanning electron microscopy (SEM) was used for high resolution inspection of micron-scale patterns.

\section{MILLIMETER-SCALE RESULTS}

\section{Contacted Cells}

The first design tested here was the square cell mask (Figure 3), in discrete-layer mode. The mask shown in Figure 8a was $0.85 \mathrm{~mm}$ thick, and had $2.8 \mathrm{~mm}$ square apertures on a $3.0 \mathrm{~mm}$ lattice. In total three depositions were used to produce the array in Figure 8b, without any translation of the sample. Two depositions were performed from azimuths pointing right $\left(0^{\circ}\right)$ and down $\left(-90^{\circ}\right)$, both using $58^{\circ}$ elevation. A further deposition was performed at $135^{\circ}$ azimuth using $66^{\circ}$ elevation. In principle all the depositions can be performed without breaking the vacuum, but in this case the chamber was opened to verify the accuracy of the stage alignment.

Polarization imaging, as shown in Figure 9, reveals differences in transmission of the different elements under different polarization conditions. In particular, the transmission of three elements oscillates under rotating polarization, with the phase indicating that the optical elements have axes at 0,45 and 90 degrees. Additionally, the overlap element (bottom right quadrant) shows minimal oscillation, consistent with low retardation. Quantitative measurements at $633 \mathrm{~nm}$ gave retardations of approximately $+80^{\circ}$ and $-110^{\circ}$ for the upper left and lower right quadrants (axes aligned to the page), with the difference in magnitude directly attributed to differences in thickness.

While the pattern in Figure 9 is clearly recognized as the desired arrangement, there are some imperfections in the pattern. Line edge roughness and finite corner radius can be attributed to corresponding features in the mask, which is simply a mask manufacturing issue. There is also some evidence of misalignment of the elements, which is a function of deposition alignment. Note that these issues are not specific to the design and will apply to all results presented here. 
For comparison, the same type of mask was tested using a single stage of adapted serial bi-deposition. In this case a different mask was used to optimize birefringence of the overlap region. This alternative square cell mask was $0.4 \mathrm{~mm}$ thick and had $3.6 \mathrm{~mm}$ apertures on a $4.5 \mathrm{~mm}$ pitch. Deposition alternated between two azimuths, one pointing right and one pointing down, with approximately 3nm per step. The elevation angle was $78^{\circ}$.

Polarized images of these elements are shown in Figure 10.

Strong retardation at $45^{\circ}$ is evident in the overlap regions, as seen in Figure 10a. As expected the two types of singleazimuth elements have approximately equal magnitude of retardation (Figure 10b) but along perpendicular planes (Figure 10c). Ellipsometry at $633 \mathrm{~nm}$ shows that the overlap element has approximately $140^{\circ}$ retardation aligned at $45^{\circ}$ and that the single-azimuth elements have $\pm 70^{\circ}$ aligned to the page. There is some evidence of retardation variation of approximately $10^{\circ}$ between the same types of elements, which may be due the sensitivity of glancing depositions to finite source effects.

\section{Suspended Sheet}

Moving now to suspended masks, the square aperture design in Figure 5 was experimentally verified. The mask shown in Figure 11a was $0.7 \mathrm{~mm}$ thick and had $2.8 \mathrm{~mm}$ square apertures on a $4.2 \mathrm{~mm}$ lattice. The mask was suspended at $h=1.05 \mathrm{~mm}$ using a $0.7 \mathrm{~mm}$ thick spacer. Serial bi-deposition was applied to optimize birefringence. In this case only two deposition stages were used to minimize processing steps. The two perpendicular SBD elements were deposited in a single deposition by using four right-angle SBD steps per turn, with an elevation angle of $63^{\circ}$. The $45^{\circ}$-azimuth elements were deposited using regular SBD, at an elevation angle of $70^{\circ}$. The resulting array is shown in Figure 11b, where very slight misalignment of the set of $45^{\circ}$-azimuth elements is evident.

Once again, polarization imaging confirms strong retardation of the $45^{\circ}$ elements and the two types of perpendicular elements, as seen in Figure 12. Ellipsometry provides additional confirmation of the opposite sign of perpendicular elements. Quantitatively, results at 633nm showed approximately $80^{\circ}$ retardation for the $45^{\circ}$ azimuth elements, and $\pm 120^{\circ}$ for elements aligned to the page. In comparison, elements that did not receive SBD had corresponding retardations of $20^{\circ}$ and $\pm 35^{\circ}$ respectively. 


\section{MICRON-SCALE RESULTS}

\section{Contacted Pillars}

The square pillar design (Figure 4) was tested using lithographic generation of the mask, which provided the opportunity to examine the scaling behavior of the general masking technique. In this case the mask was produced using $1.4 \mu \mathrm{m}$ of photoresist (Shipley S-1813) on glass. The pillars were $5 \mu \mathrm{m}$ wide on a $10 \mu \mathrm{m}$ lattice, as seen in Figure 13.

Approximately $0.6 \mu \mathrm{m}$ of silicon was deposited, using SBD with two perpendicular azimuths aligned to the mask, and an elevation angle of $76^{\circ}$. Inspection by polarizing microscope confirmed the expected properties. Rotation of the crossed polarizers (Figure 14, a and b) revealed that the overlap element had significant birefringence along the $45^{\circ}$ azimuth. Insertion of a dispersion retarder (tint plate) in Figure 14c showed a color difference between the perpendicular elements, consistent with a difference in the sign of the birefringence. Ellipsometry of larger regions confirmed these results, and showed an absolute retardation of approximately $15^{\circ}$ at $633 \mathrm{~nm}$ for each of the three elements.

Investigation with field emission SEM (Figure 15) shows that the elements are not rectangular, due to the shadow cast by the overcut resist profile. The desired vertical side walls could be achieved by using a more precise process, such as dry-etching, to produce the mask. Other imperfections of the pattern are evident. The lateral broadening of the blank element is due to the space occupied by sidewall growth, and could be partially ameliorated by reducing the resist pillar width, but this is dependent on the film thickness. The tapering of thickness in the transitions between elements is due to incremental shadowing from growth on the mask, and is probably inevitable. Additional bulk selfshadowing due to different film growth rates is also a likely contributor to non-ideal behavior. In general, the resolution of the masking technique appears to be limited by the secondary perturbations of growth induced by the mask, which produce unplanned areas with widths a few times the coating thickness.

\section{CONCLUSION}

We have demonstrated a masking technique for producing arrays of nano-structured elements. Note that while we have focussed on optical polarizing properties, the deposited elements have other tensor properties that may be useful. All designs that were tested successfully generated the required polarizing patterns, and issues that contribute to nonideal patterning behavior have been identified. Some of these issues can be controlled, by ensuring the accuracy of 
both mask fabrication and deposition alignment. When the technique is applied to miniature scales, some degree of deviation is expected. We suggest exclusion of non-conforming regions as a realistic and simple solution suitable for some applications, such as imaging polarimeters. Overall, the technique is useful because it can be applied to many materials, expensive lithography equipment is not required, and multiple properties can be generated in a single deposition step.

\section{ACKNOWLEDGEMENTS}

This work was supported by the New Zealand New Economy Research Fund (contract UOCX-0211). We gratefully acknowledge the skill of Mr Peter Simpson in producing the millimetric masks. 


\section{REFERENCES}

T. Motohiro and Y. Taga, Applied Optics 28, 2466-2482 (1989).

R. Messier and A. Lakhtakia, Materials Research Innovations 2, 217-222 (1999).

K. D. Harris, A. Huizinga, and M. J. Brett, Electrochemical and Solid State Letters 5, H27-H29 (2002). Publishing Co. Pte. Ltd., 1997).

I. Hodgkinson and Q. H. Wu, Applied Physics Letters 74, 1794-1796 (1999).

R. M. A. Azzam, Applied Physics Letters 61, 3118-3120 (1992). M. Malac, R. F. Egerton, M. J. Brett, and B. Dick, Journal of Vacuum Science \& Technology B 17, 26712674 (1999).

I. Hodgkinson, Q. H. Wu, M. Arnold, and R. Blaikie, Microelectronic Engineering 57-8, 833-836 (2001).

K. D. Harris, K. L. Westra, and M. J. Brett, Electrochemical and Solid State Letters 4, C39-C42 (2001).

S. R. Kennedy, M. J. Brett, O. Toader, and S. John, Nano Letters 2, 59-62 (2002).

M. Seto, K. Westra, and M. Brett, Journal of Materials Chemistry 12, 2348-2351 (2002).

M. W. Horn, M. D. Pickett, R. Messier, and A. Lakhtakia, Nanotechnology 15, 303-310 (2004).

J. P. Guo and D. J. Brady, Optical Engineering 36, 2268-2271 (1997).

J. P. Guo and D. Brady, Applied Optics 39, 1486-1492 (2000).

C. K. Harnett and H. G. Craighead, Applied Optics 41, 1291-1296 (2002).

G. P. Nordin, J. T. Meier, P. C. Deguzman, and M. W. Jones, Journal of the Optical Society of America A 16, 1168-1174 (1999).

T. Hamamoto, H. Toyota, and H. Kikuta, Proceedings of the International Society for Optical Engineering 4440, 293-300 (2001).

B. Schnabel, E. B. Kley, and F. Wyrowski, Optical Engineering 38, 220-226 (1999).

9 G. J. Dolan, Applied Physics Letters 31, 337-339 (1977).

M. D. Arnold, PhD Thesis, University of Otago, 2005. 


\section{LIST OF FIGURES}

Figure 1: An example mask. The plan view (a) shows the arrangement of azimuths. The mask is shown as black and the substrate is grey. Global evaporation azimuths are indicated by the large white arrows, whereas local deposition azimuths are shown by small black arrows. The section view (b) shows the shadowing condition on the elevation angle $\theta$.

Figure 2: Diagram of oblique vapor deposition, showing in particular the deposition angles $\theta$ (elevation) and $\xi$ (azimuth).

Figure 3: Design for square cell contact mask, with plan (a) and section (b). The dotted arrows represent the direct method for deposition of a $45^{\circ}$ retarder azimuth. Alternatively, SBD can be used to generate the required element in the overlap region.

Figure 4: Design for square pillar contact mask, with plan (a) and section (b). The mask height shown is the minimum.

Figure 5: Design for square suspended aperture mask, with plan (a) and section (b).

Figure 6: Three-fold symmetric suspended masks: (a) rhombus aperture sheet, (b) hexagon aperture sheet, (c) hexagon cells suspended.

Figure 7: Three-fold symmetric contacted -cell masks, with hexagons (a) and triangles (b).

Figure 8: Verification of discrete layer square cell design, showing the mask (a) and the deposited array (b) imaged without polarizers.

Figure 9: Images of four elements from the square cell discrete design, between parallel polarizers with a compensating quarter-wave plate at $45^{\circ}$. The series shows rotating polarizers, the direction of the compensator being indicated by the white arrows.

Figure 10: Optical images of elements deposited through a square cell mask using adapted SBD. Frames (a) and (b) use crossed polarizers aligned with the page and at $45^{\circ}$ respectively. Frame (c) has parallel polarizers aligned at $45^{\circ}$ plus a quarter-wave plate at $0^{\circ}$.

Figure 11: Verification of square aperture suspended mask, showing the mask (a) and deposited elements (b). 
Figure 12: Optical images of the elements deposited using the suspended mask. Polarizers were crossed, aligned with the page in (a) and at $45^{\circ}$ in (b).

Figure 13: Image of resist mask, at an elevation angle of $60^{\circ}$. Note overcut resist profile. The pillar height is approximately $1.4 \mu \mathrm{m}$.

Figure 14: Optical images of the microscopic elements, with the sample between crossed polarizers. Image (a) has polarizers aligned to the page, and in (b) the polarizers are rotated to $45^{\circ}$. In (c) an additional dispersion retarder has been inserted.

Figure 15: Plan electron micrograph of the deposited "square-pillar” array. Deviation from ideal behavior is evident as a result of lateral growth on the mask. 
Figure 1, Arnold et al, JVSTB
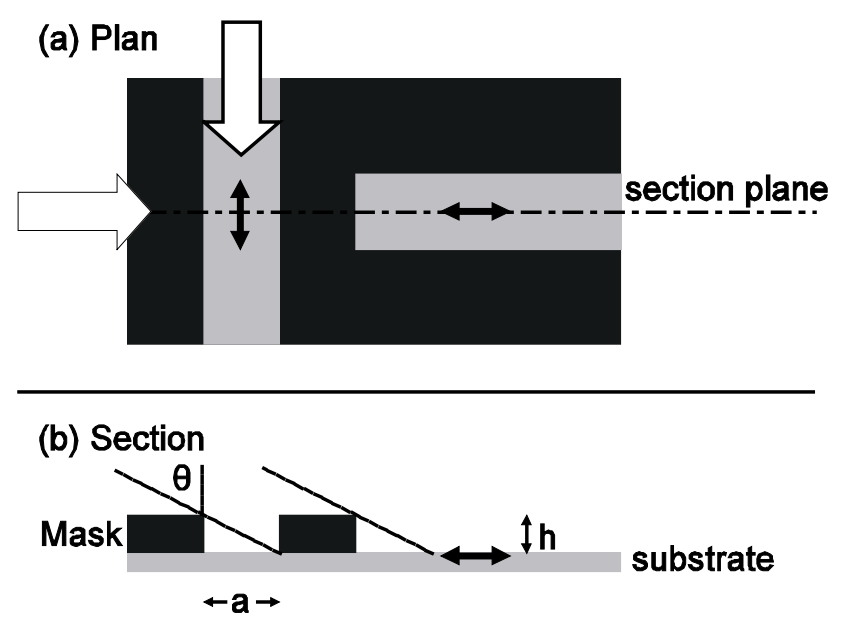
Figure 2, Arnold et al, JVSTB

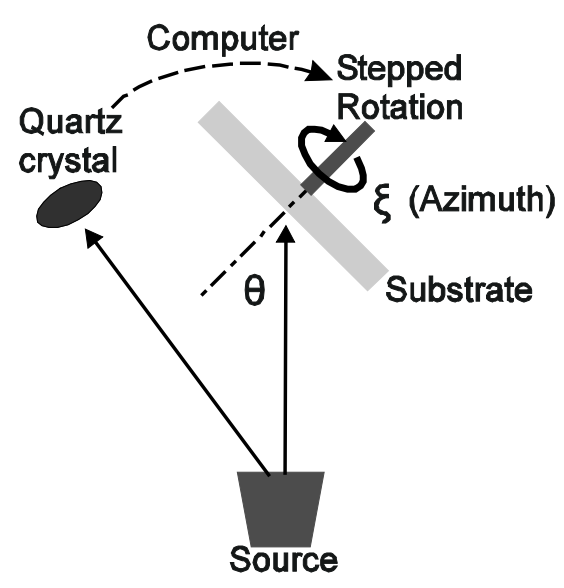


Figure 3, Arnold et al, JVSTB

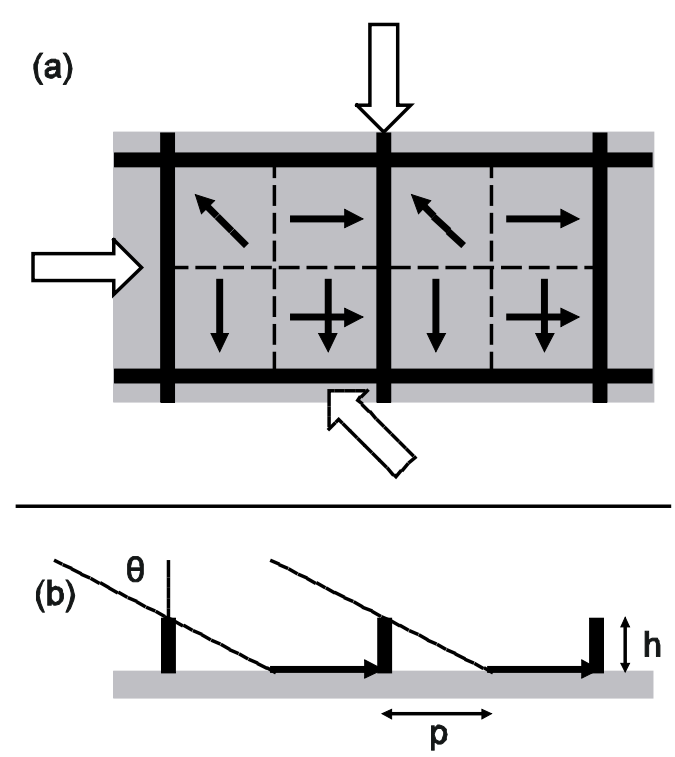


Figure 4, Arnold et al, JVSTB

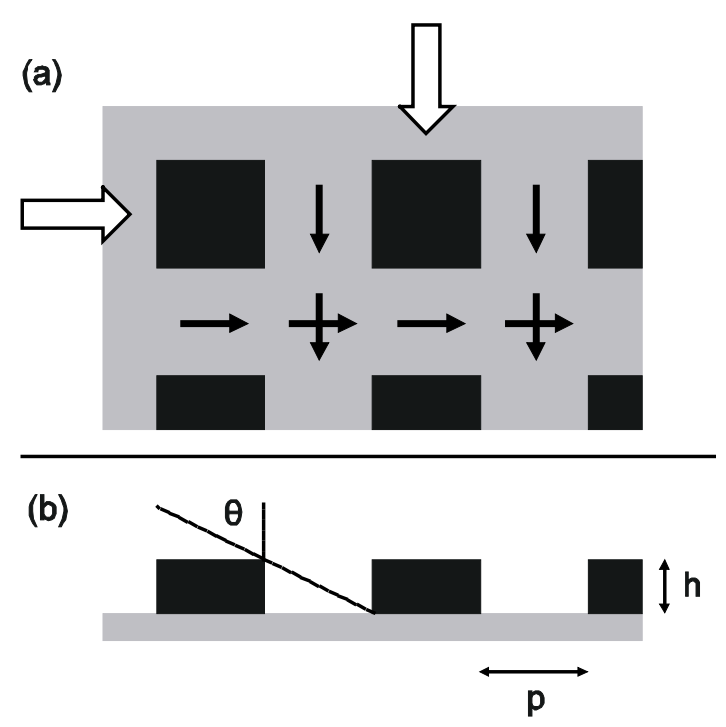


Figure 5, Arnold et al, JVSTB
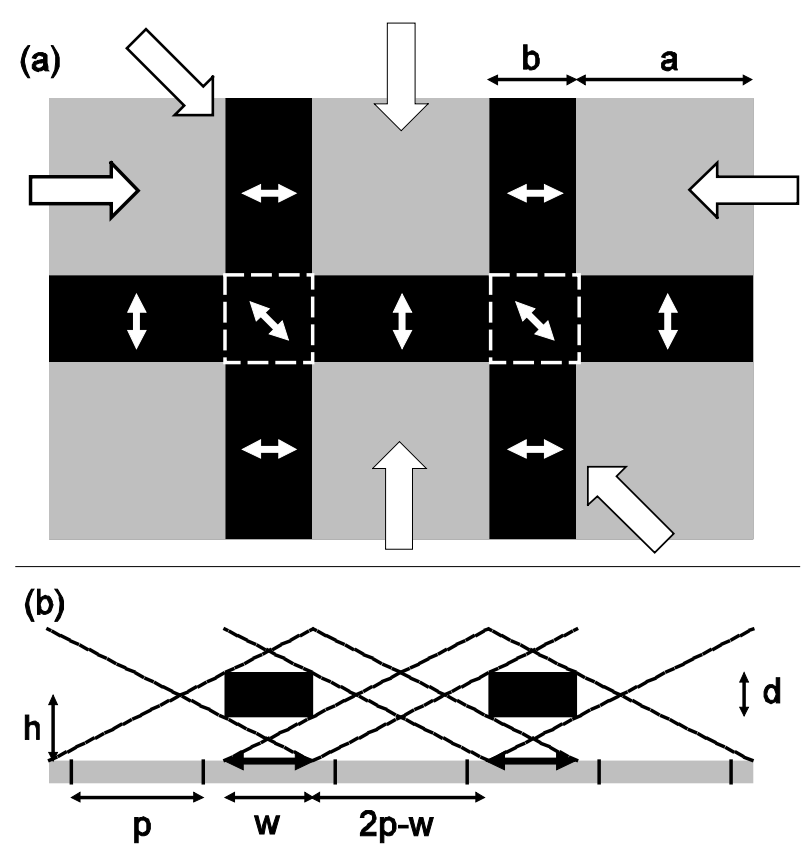
Figure 6, Arnold et al, JVSTB

(a)
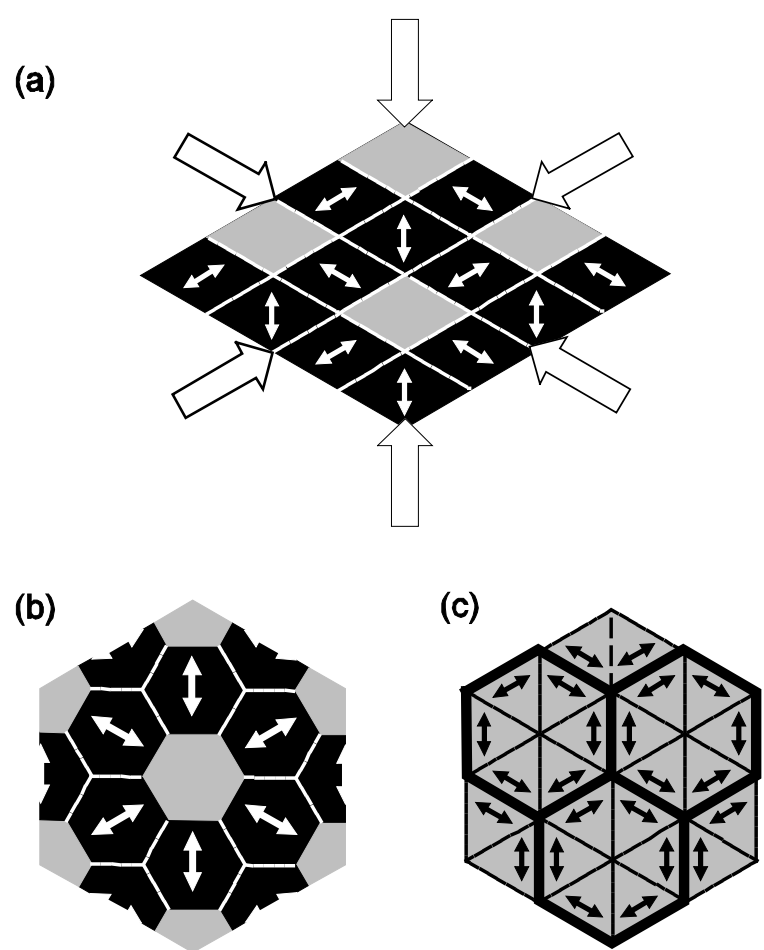
Figure 7, Arnold et al, JVSTB

(a)

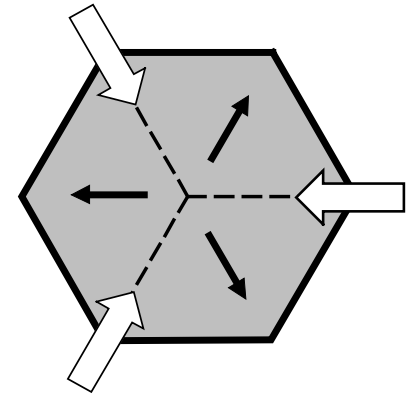

(b)

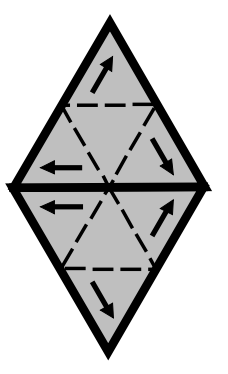


Figure 8, Arnold et al, JVSTB
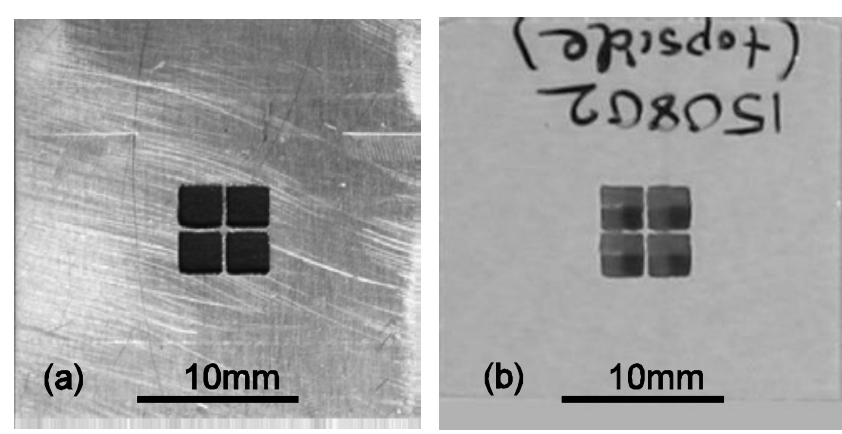
Figure 9, Arnold et al, JVSTB

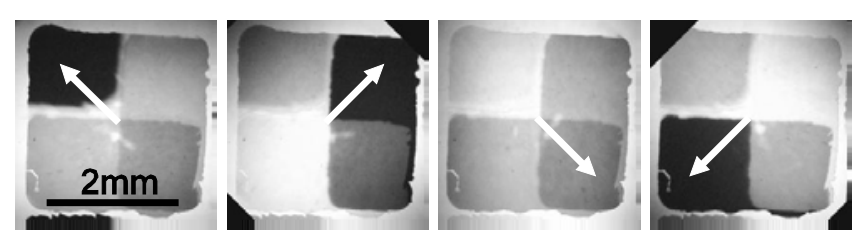


Figure 10, Arnold et al, JVSTB
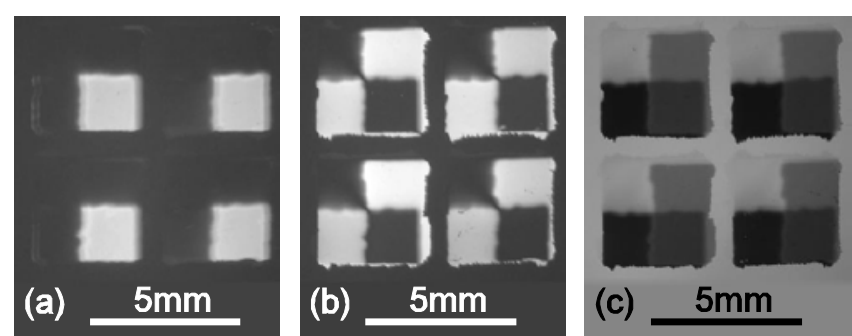
Figure 11, Arnold et al, JVSTB
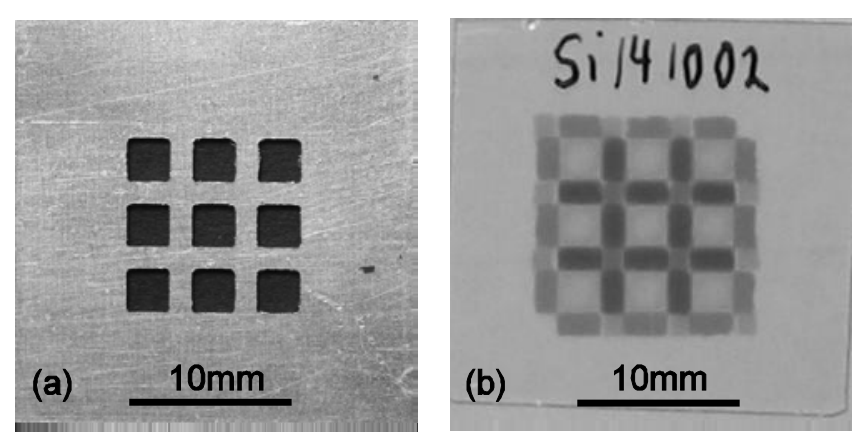
Figure 12, Arnold et al, JVSTB

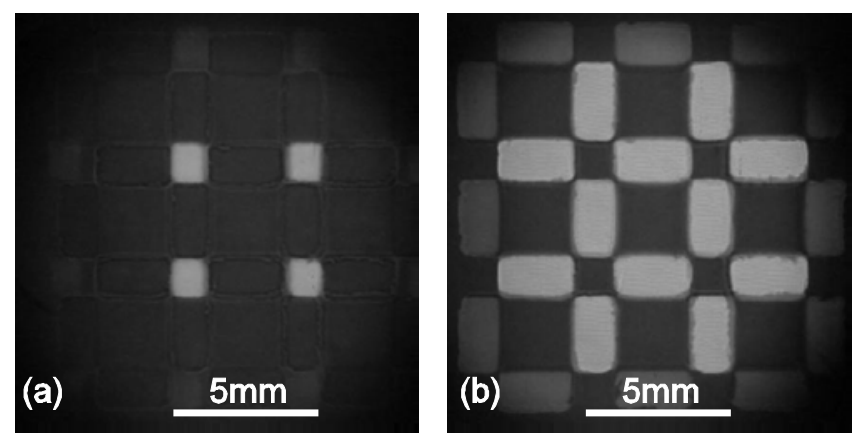


Figure 13, Arnold et al, JVSTB

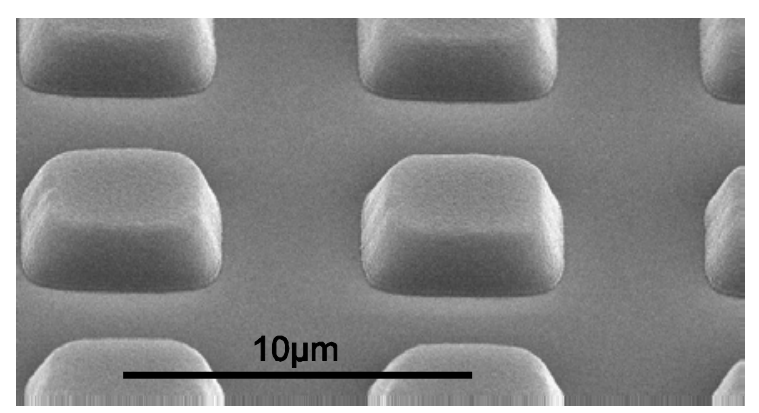


Figure 14, Arnold et al, JVSTB

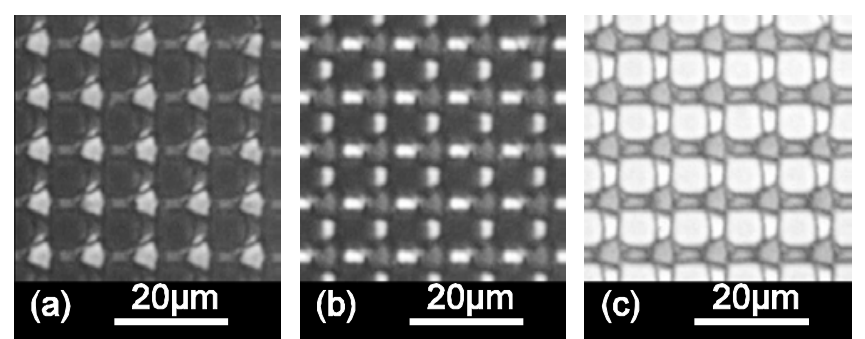


Figure 15, Arnold et al, JVSTB

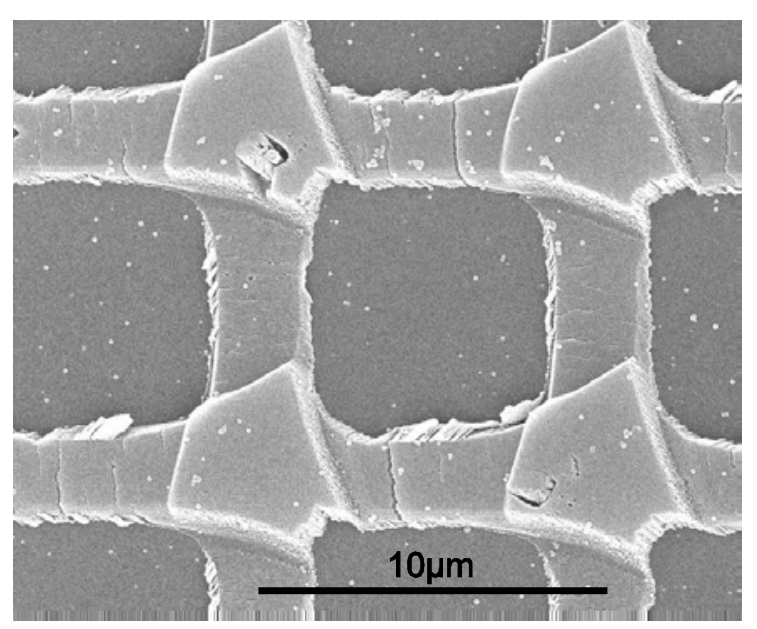

